\title{
Does Origins of Life Research Rest on a Mistake?
}

\author{
Roger WHITE \\ Massachusetts Institute of Technology
}

\section{Origins of Life Research}

Contemporary scientific research on the origins of life is marked by the following features.

(1) There are a wide variety of suggestions as to how life might first have emerged.

This disagreement extends to the fundamental details of physical and biochemical theories. On the other hand,

(2) There is almost universal agreement that life did not first come about merely by chance.

This is not to say that all scientists think that life's existence was inevitable. The common view is that given a fuller understanding of the physical and biological conditions and processes involved, the emergence of life should be seen to be quite likely, or at least not very surprising. The view which is almost universally rejected by researchers in the field is that the numerous and prima facie improbable physical and biological requirements for life all fell together just by a fluke, like so many dice tumbling out of a bag and landing all sixes.

Most importantly, for the purposes of the following discussion,

(3) The conviction that life did not arise largely by chance is treated as epistemically prior to the development of alternative theories. 
It is not that theorists deny that life arose by chance because, as it happens, they have a better and more plausible story to offer (although of course many theorists do, perhaps rightly, take themselves to have such). Rather, an understanding of what is required for there to be living creatures is what convinces scientists that life didn't just arise by chance. It is this conviction that is the impetus for the active research program into life's origins. It appears that if those theorists who do endorse a particular non-chancy explanation of life's origin had to abandon their view in the light of new evidence, they would typically still retain the conviction that life did not just arise by chance. Given what we know about the requirements for life such as the structure of the DNA/protein machinery required for evolution, too "chancy" an explanation of life's origin is thought to be theoretically unacceptable.

And lastly,

(4) The suggestion that the origin of life might be due to any kind of purposeful agency is not considered as a serious option, and does not play any explicit role in theorizing.

There are always odd exceptions of course, but they tend to appear in scientists' popular writings. The kind of explanations of life's emergence that scientists look for appeal to ordinary physical properties, forces, and laws, having nothing to do with the purposes of any agent.

I will not be taking issue with any of these features individually. But I will raise doubts about how (3) and (4) can sensibly hang together. What puzzles me is why, if appeals to intelligent agency are not on the table, we should be so reluctant to attribute the origin of life largely to chance. My purpose is to question a common approach to the subject of life's origin. Very roughly, this approach consists in an aversion to appeals to chance in accounting for life's origin prior to an evaluation of alternative hypotheses. I hope to make the issue clearer in the following sections. But let me be clear at the outset about the aim and scope of this paper. It is not my purpose to evaluate specific scientific proposals on the origin of life. My discussion will be very abstract, not entering into any of the details of cutting edge research. The reason for this, as I hope will become clear, is that my concern is with an abstract epistemological question which arises prior to detailed investigation, and does not hinge on the details of research.

We should first get a little clearer on the issue, by way of some analogies.

\section{Three Pebble Patterns}

Some states of affairs are quite reasonably attributed to chance.

Pebble Pattern 1: Pebbles are scattered in a disorderly fashion as we typically find them on the sidewalk. 
If someone were to ask why the pebbles fell in this specific pattern, we should be satisfied with the response "They just fell that way by chance. A car wheel kicked up the stones and they happened to land this way." Of course it is highly improbable that a random scattering of pebbles should result in precisely this arrangement on the path, but this is no reason to doubt that their arrangement was due to anything but chance. Random improbabilities are commonplace - people win lotteries, hands of cards are dealt, and so on-without giving us any reason to suspect that there is a deeper reason for their occurrence than blind chance.

Of course there might be a more interesting explanation for the arrangement of these pebbles. Perhaps they were placed there deliberately to form a message in a secret code, or perhaps there are certain physical laws and properties of pebbles, either unknown or whose consequences are unappreciated, which render it likely that even if the pebbles are tossed at random they will arrange themselves in this very pattern. Moreover, there is nothing to stop us discovering that some such non-chancy explanation is correct. But while explanations of this kind are possible, we have no reason to pursue them. It is not just that they are far fetched - even if we are very open minded about these suggestions, the fact that the pebbles are arranged as they are gives us no reason at all to suppose that there is any explanation for their arrangement other than chance. This arrangement of pebbles is one of those states of affairs for which the search for a deeper explanation than blind chance is inappropriate.

Not so for all states of affairs.

Pebble Pattern 2: At the English seaside, pebbles cover the beach in descending order of size toward the shoreline.

No one supposes that it is just a fluke that these pebbles are arranged in this order, as if they had just fallen off the back of a dump truck. The correct explanation presumably has something to do with the lawful correlations between physical properties such as volume, mass, and inertia (my rough guess is that as the waves and tides wash in and out, the smaller stones being lighter are more easily swept back to the shoreline). But even if we know next to nothing about the physics involved, the simple regularity of the pattern suggests that these pebbles didn't get that way by accident. Prior to formulating any specific alternative explanation we can rightly judge that this arrangement is not simply due to chance. This may be the impetus for a search for the correct explanation.

Pebble Pattern 3: The pebbles are arranged to form a stick figure with a smile on its face.

We would likewise have a hard time believing that these pebbles got this way by chance. There can be little doubt about the general form of the correct 
explanation here, namely that the positioning of the pebbles was influenced on purpose by an agent. Perhaps a full explanation would require more details as to who arranged the pebbles and how. But their being arranged as they are is enough to make us suspect that some explanation along these lines is correct, and perhaps motivate us, if we are interested, to find the fuller explanation.

\section{Chance}

It is important to avoid a certain misunderstanding about the kind of distinction that is invoked by this talk of chance. Sometimes the term 'chance' is reserved for processes that are non-deterministic and irreducibly probabilistic. This narrow sense of the term is not what is at issue here. Perhaps determinism is true or at least approximately correct at the macroscopic level. In this case any arrangement of matter is the inevitable result of certain prior conditions given the physical laws. Nevertheless, there is an important sense of 'chance' in which we might still speak of games of chance such as roulette, where the winner is said to win by chance. By chance I might run into an old friend I haven't seen in years. Not so long ago, scientists suggested that the very earliest living organism was the result of a "chance collision of molecules" in a pre-biotic soup, where this was not meant to be incompatible with determinism. I think we have a good enough grip on what they had in mind: some simple molecules were shuffling about in the soup - much like shaking Lego pieces in a box - until they just happened to form a stable structure capable of reproduction. It is this kind of view that is being denied when contemporary theorists insist that life did not originate by chance.

We can understand a little better what is at issue by considering the different kinds of explanation we are inclined to give the three pebblearrangements. When we deny that Pebble Patterns 2 and 3 were merely the result of chance, we are supposing that there are certain facts - even if we have only the dimmest idea what these facts might be - that help explain the arrangement of the pebbles, and given which the arrangement is to be expected. But it is not just any kind of explanatory fact that we have in mind. For as we have noted, if determinism holds, even Pebble Pattern 1 is highly probable given certain antecedent conditions. What makes the explanation that we expect for Patterns 2 and 3 different from that expected for Pattern 1 is a kind of robustness or stability. Given the explanatory facts - say, that larger objects are heavier and consequently harder to move, or that Sydney was making a portrait of her dad-something like patterns 2 and 3 are to be expected even given wide variation in the initial conditions. By contrast, if Pattern 1 was just the result of a car tire skidding on gravel, the arrangement of the pebbles was extremely sensitive to the precise initial conditions. Had any one of a number of factors such as the prior position of the pebbles or the speed and angle of the car tire differed even slightly, the pebbles would 
have fallen in a very different pattern. Not so with Patterns 2 and 3. Given the intentions of the pebble-artist, no matter what state the pebbles started out in, we should expect them to end up arranged to form a stick-figure. Similarly with the size-ordered pebbles of pattern 2 . The motion of the ocean on the beach should eventually sift any pile of pebbles into such a pattern, no matter how they were first arranged. This is part of what makes the latter explanations so satisfying. It would be deeply unsatisfying to account for patterns 2 and 3 only by appeal to a very specific and precarious set of initial conditions. It is an explanation of the more stable sort-one that does not render the phenomena highly sensitive to precise and highly improbable initial conditions - that scientists are seeking when they find it unacceptable to suppose that life arose by chance. ${ }^{1}$

\section{Chance and the Origin of Life}

What concerns us here is not the evolution of biological complexity and diversity from simpler organisms - we can suppose that a broadly Darwinian account takes care of that-but rather how it all got started in the first place. Of course it is hard to say at what point a molecular system counts as being alive. To sharpen our discussion we can focus on the emergence of a selfreplicating system with the capabilities required for the familiar Darwinian process to get going. On the standard Darwinian account, evolution began with the existence of some molecular machinery capable of manufacturing systems similar to itself containing the information to build further copies, and so on. Given such a system, a process of random mutation and natural selection can lead to all manner of variations.

Our concern is with the extent to which it is acceptable to appeal to chance in our account of how life emerged. Here I am going to indulge in a simplification. Various proposals about the origin of life appeal to chance to a greater or lesser degree, and appeal to it more in some parts of the theory than in others. I will be speaking somewhat simplistically in terms of whether life is the result of chance, or if some non-chancy explanation is correct. While this might not do justice to all the subtleties of the research, it will do for our purposes.

It is said to be extremely improbable for life to come about by chance. There are at least two sources for this apparent improbability. First, the existence of life appears to depend on the extremely fine adjustment of various physical parameters. Had the values of various fundamental constants differed ever so slightly, we would not have had anything like a stable universe in which any kind of life could evolve. If we could wind back the universe to the big bang, our confidence that any kind of life would result should be close to zero. ${ }^{2}$ Second, the conditions required for a process of natural selection to get going turn out to be more difficult to meet than had been imagined. Increased understanding of the immense complexity of the 
molecular machinery involved, together with a greatly reduced estimate of the time window available for its random formation has led scientists to claim that the probability of a self-replicating system arising by chance is incredibly low. These claims can be questioned in various ways, but as they are widely held by leading researchers in the field, let us grant that the probability of life arising by chance is extremely low and consider if this has any implications for the acceptability of appeals to chance in accounts of the origin of life.

I will be drawing some parallels with the cosmological issues, but the main concern of this paper is the molecular origins of life. The following appears to be a fair representation of the attitudes of most scientists working on the origin of life in the last few decades:

We now know that the probability of life arising by chance is far too low to be plausible, hence there must be some deeper explanation that we are yet to discover, given which the origin of life is at least reasonably likely. Perhaps we have little idea yet what form this explanation will take - although of course it will not appeal to the work of a rational agent; this is would be a desperate last resort, if an option at all-but we have every reason to look for such an explanation, for we have every reason to think there is one.

In a detailed survey of the field, Iris Fry $(1995,2000)$ argues that although the disagreements among origin of life theorists run very deep, relating to the most basic features of the models they propose, the view sketched above is a fundamental unifying assumption (one which Fry strongly endorses). Some researchers in the field are even more optimistic of course. They believe that they have already found the explanation, or at least have a good head start on it. But their commitment to the thesis above is epistemically more basic, in the sense that it motivated their research in the first place and even if their theories were shown to be false, they would retain this basic assumption. ${ }^{3}$

There is a very small group of detractors, whom Fry (1995) calls the "Almost a Miracle Camp" including Francis Crick (1981), Ernst Mayr (1982), and Jaques Monod (1974), who appear to be content with the idea that life arose by chance even if the probability of this happening is extremely low. ${ }^{4}$ According to Crick "the origin of life appears at the moment to be almost a miracle, so many are the conditions which would have had to been satisfied to get it going" (1981: 88); the emergence of life was nevertheless a "happy accident" (p. 14). ${ }^{5}$ According to Mayr, "a full realization of the near impossibility of an origin of life brings home the point of how improbable this event was." (1982: 45). Monod famously claimed that although the probability of life arising by chance was "virtually zero... our number came up in the Monte Carlo game" (1974: 137). Life, as Monod puts it, is "chance caught on a wing" (p. 78). That is, although natural selection took over early to produce the diversity of life, its origin was nothing but an incredibly improbable fluke. 
However, the vast majority of experts in the field clearly define their work in opposition to this view. The more common attitude is summed up neatly by J. D. Bernal.

[T] he question, could life have originated by a chance occurrence of atoms, clearly leads to a negative answer. This answer, combined with the knowledge that life is actually here, leads to the conclusion that some sequences other than chance occurrences must have led to the appearances of life. (quoted in Fry 2000: 153)

Having calculated the staggering improbability of life's emergence by chance, Manfred Eigen (1992) concludes,

The genes found today cannot have arisen randomly, as it were by the throw of a dice. There must exist a process of optimization that works toward functional efficiency. Even if there are several routes to optimal efficiency, mere trial and error cannot be one of them. (p. 11)

It is from this conclusion that Eigen motivates his search for a physical principle that does not leave the emergence of life up to blind chance, hence making it reproducible in principle:

The physical principle that we are looking for should be in a position to explain the complexity typical of the phenomena of life at the level of molecular structures and syntheses. It should show how such complex molecular arrangements are able to form reproducibly in Nature. (p. 11)

According to Christian de Duve (1991),

....unless one adopts a creationist view,... life arose through the succession of an enormous number of small steps, almost each of which, given the condition at the time had a very high probability of happening. ..the alternative amounts to a miracle...were [the emergence of life] not an obligatory manifestation of the combinatorial properties of matter, it could not possibly have arisen naturally. (p. 217)

Not all theorists follow De Duve so far as suggesting that life's emergence must be inevitable. While not a specialist in the area, Richard Dawkins (1987) captures the attitude that appears to dominate scientific research into life's origin. According to Dawkins,

All who have given thought to the matter agree that an apparatus as complex as the human eye could not possibly come into existence through [a single chance event]. Unfortunately the same seems to be true of at least parts of the apparatus of cellular machinery whereby DNA replicates itself (p. 140) 
In considering how the first self-replicating machinery arose, Dawkins asks "What is the largest single event of sheer naked coincidence, sheer unadulterated miraculous luck, that we are allowed to get away with in our theories, and still say that we have a satisfactory explanation of life?" (p. 141) And he answers that there are strict limits on the "ration of luck" that we are allowed to postulate in our theories. ${ }^{6}$ According to Dawkins, an examination of the immense complexity of the most basic mechanisms required for DNA replication is sufficient to see that any theory which makes its existence a highly improbable fluke is unbelievable, quite apart from what alternative explanations are on the table.

\section{When Should we Doubt that Something was due to Chance?}

What interests me is just why the 'Almost a Miracle' camp is so small. Why is it that the vast majority of researchers in the field agree with Dawkins that we cannot credibly suppose that life arose by spontaneous random generation if the chance of this happening was extremely small. In this section I want to consider on what grounds in general one should reject an explanation that appeals to improbable chance events. The answer will be applied to the case of life's existence, but I will continue to illustrate the ideas in terms of the pebbles.

We can roughly divide up the possible ways that pebbles can be arranged in a specified area into a set of elementary possibilities. For instance we might put a grid over the area and distinguish possible arrangements according to which cells of the grid are occupied. The state of affairs $\mathrm{S}$ that we are concerned with (e.g., that the pebbles form a stick-figure) obtains if and only if some member of a certain subset of the elementary possibilities obtains. Call the hypothesis that the process that resulted in $\mathrm{S}$ was just a matter of chance, the Chance Hypothesis $\mathrm{C}$. This means roughly that the process was not biased toward some elementary possibilities over others. On the Chance Hypothesis, the elementary possibilities are considered about equally probable.

Now there are two errors to be avoided when thinking about extremely low probabilities. The first is to suppose that the extreme improbability of a chance process resulting in a certain state of affairs is a reason by itself to doubt that this state of affairs was the result of chance. The mistake may lie in the conflation of the two claims: that it is improbable that chance would result in $\mathrm{S}$, and that it is improbable that $\mathrm{S}$ is the result of chance. The first is to say that the conditional probability $\mathrm{P}(\mathrm{S} \mid \mathrm{C})$ is low, while the second says that the converse, $\mathrm{P}(\mathrm{C} \mid \mathrm{S})$ is low. The latter does not follow from the former. For any blade of grass, the probability that a random swing at a golf ball will result in that blade being hit is very low. It does not follow that for whichever blade of grass is hit, it is improbable that this hitting is the result of a random swing. 
The second mistake in thinking about low probabilities is an overreaction to the first. It is to dismiss any doubts that something was due to chance simply on the grounds that something had to happen, and whatever did happen was bound to be highly improbable. ${ }^{7}$ Clearly there are some states of affairs that do cry out for a non-chancy explanation, for which it would be foolish to accept as a mere coincidence. Our earlier examples of the pebbles falling in Patterns 2 and 3 serve to illustrate this. But any number of examples can be invented: a monkey types a sonnet, one person wins ten lotteries in a row, a die lands on 6 fifty times in a row. All of these could have come about by chance, but it is highly implausible that they did.

So when is it reasonable or unreasonable to appeal to chance in our explanations? I will be taking a broadly Bayesian approach to this question, but not one that appeals to anything too controversial. Rather than give fully general conditions for rationally rejecting the Chance Hypothesis, we need only focus on the following theorem that gives a necessary condition for the state of affairs $\mathrm{S}$ to raise any doubt about $\mathrm{C}$.

T1: $\mathrm{P}(\mathrm{C} \mid \mathrm{S})<\mathrm{P}(\mathrm{C})$ only if $\mathrm{P}(\mathrm{S} \mid \mathrm{C})<\mathrm{P}(\mathrm{S} \mid \sim \mathrm{C})$

That is, $\mathrm{S}$ disconfirms (makes less probable) the Chance Hypothesis only if $\mathrm{S}$ was less to be expected given that the process that led to $\mathrm{S}$ was a matter of chance, than given that it was not.

Now to deny the Chance Hypothesis is to claim that the process that resulted in $\mathrm{S}$ was biased in some way-that the conditions were such that some of the elementary possibilities were objectively more likely to obtain than others. How are we to evaluate the probability $\mathrm{P}(\mathrm{S} \mid \sim \mathrm{C})$ ? There are any number of different ways in which the process leading to $S$ might have been biased, some which (strongly or weakly) favor $\mathrm{S}$ and others that disfavor it. To deny $\mathrm{C}$ is simply to claim that the process was biased in some way or other, so it needn't be obvious how likely $\mathrm{S}$ is given $\sim \mathrm{C}$.

One way to attack this problem is to partition $\sim \mathrm{C}$ into a set of more specific bias-hypotheses $\left\{B_{1}, B_{2}, \ldots, B_{n}\right\}$ each one giving more details about the way in which the process was biased. We can then appeal the following theorem:

T2: $\mathrm{P}(\mathrm{S} \mid \sim \mathrm{C})=\sum_{\mathrm{i}} \mathrm{P}\left(\mathrm{S} \mid \mathrm{B}_{\mathrm{i}}\right) \mathrm{P}\left(\mathrm{B}_{\mathrm{i}}\right)$

If the bias-hypotheses $B_{1}-B_{n}$ are specific enough, there will be less difficulty in assigning a value to $\mathrm{P}\left(\mathrm{S}_{\mathrm{S}} \mathrm{B}_{\mathrm{i}}\right)$ for each $\mathrm{i}$. But this will shift the problem to assigning a reasonable probability distribution over $B_{1}-B_{n}$. What we need is a partition of $\sim \mathrm{C}$ specific enough to give some guidance in assigning likelihoods but not so specific as to create difficulties in assigning a distribution over the partition. 
I think a good place to start is by partitioning $\sim \mathrm{C}$ into the hypotheses of what I will call intentional and non-intentional biasing. A process such as pebble arranging is intentionally biased if certain elementary possible outcomes are more likely than others due to the purposeful action of some agent. By "due to the purposeful action" of an agent, of course I mean to exclude cases in which the outcome is just some unforeseen consequence of an agent's actions. The idea is that the agent desires that a certain state of affairs obtain and acts in order to help bring it about. A process is non-intentionally biased if this biasing is not due in any way to the purposes and actions of any agent, but rather say, the impersonal laws of nature together with properties of matter and the structure of certain physical mechanisms.

Let $\mathrm{B}_{\mathrm{I}}$ be the hypothesis that the process that led to $\mathrm{S}$ was intentionally biased in some way or other, and $\mathrm{B}_{\mathrm{N}}$ be that it was non-intentionally biased. The following theorem gives a necessary condition on S's disconfirming $\mathrm{C}$.

T3: $\mathrm{P}(\mathrm{C} \mid \mathrm{S})<\mathrm{P}(\mathrm{C})$ only if either $\mathrm{P}\left(\mathrm{S} \mid \mathrm{B}_{\mathrm{I}}\right)>\mathrm{P}(\mathrm{S} \mid \mathrm{C})$ or $\mathrm{P}\left(\mathrm{S} \mid \mathrm{B}_{\mathrm{N}}\right)>\mathrm{P}(\mathrm{S} \mid \mathrm{C})$

In considering whether the outcome $\mathrm{S}$ gives us any reason to doubt that it came about by chance, we must ask whether $\mathrm{S}$ was more to be expected on the assumption that the process from which it resulted was intentionally biased, than if it was unbiased, or if it was more to be expected given that the process was non-intentionally biased, than if it was unbiased.

We can illustrate T3's application with respect to the pebbles again, starting with the beach pebbles of Pattern 2 . That these should be arranged roughly in order of size seems more likely on the assumption that the process shuffling the pebbles was non-intentionally biased, than if they just fell on the beach by chance. This is roughly because of the regularity that they display. If the pebbles are tossed about in such a way that each is about as likely to land in one place as another, it is very unlikely that they will end up in any kind of simple regular pattern. Suppose instead that the attributes of the pebbles interact with the physical laws in such a way as to make certain arrangements more likely than others. While on this modest assumption we can't predict exactly how the pebbles will end up, simple correlations between physical parameters such as size and location are the kind of phenomena we should expect to find.

The stick-figure arrangement of Pattern 3 is highly improbable on the Chance Hypothesis. But when we consider its probability on the assumption that the arrangement of pebbles was intentionally biased, it is clearly much higher. The reason is roughly that the stick figure is one of a small class of interesting patterns, and if an agent was to go to the trouble of influencing the way the stones are arranged, there is a good chance that she would arrange them in an interesting way.

That the pebbles are arranged as in Pattern 1 is also highly improbable on the Chance Hypothesis. But in this case their arrangement seems no more 
probable on the denial of this hypothesis. There is nothing to suggest that this outcome is any more likely given that an agent influenced the arrangement of the pebbles. The arrangement just doesn't stand out as the kind of pattern that an agent might prefer. Indeed their arrangement seems less likely on the assumption of intentional biasing. Nor does the arrangement seem any more likely on the assumption of non-intentional biasing. There is nothing about this pattern of pebbles that suggests that it was any more likely to come about given that there was some physical mechanism at work that rendered some patterns more likely than others. Since the arrangement of pebbles in Pattern 1 is no more likely on the assumption of either intentional or non-intentional biasing than it is on the Chance Hypothesis, their being arranged this way gives us no reason at all the doubt that they fell this way by chance.

\section{Intentional Biasing for Life?}

If life's existence is so incredibly improbable on the Chance Hypothesis, we must ask whether it is any more likely on the denial of the Chance Hypothesis, starting first with the possibility of intentional biasing. Many have thought it to be considerably more likely. After all, living creatures are rather special, far more interesting than a universe containing nothing but hydrogen. If we were to guess whether a certain big bang was going to turn out a universe containing living creatures rather a homogenous cosmic soup, we would be more inclined to expect the first outcome if we knew that the physical parameters were being set by an agent capable of making rational choices.

Or so it has seemed to many. The line of reasoning above faces the following objection which I will call the Preference Problem: In the case of the pebbles arranged on the sidewalk to form a stick-figure, we are epistemically well placed to judge what the preferences of an agent would most likely be. We know roughly how an agent is likely to arrange pebbles because we know that if there is such a pebble-arranger, she is probably a human much like us, and we have plenty of experience to go on to know the kinds of patterns which might be found interesting or useful to agents like us. But when it comes to the big bang, or the molecular origins of life on earth, any agent who could have a hand in such matters would have to be vastly more powerful and intelligent than anything with which we are familiar. Consequently, we can have no clue as to how such a being is likely to act. We can dream up any number of possible preferences for such an agent, but we have no principled way of telling that one is more likely than any other. So the emergence of life cannot reasonably be judged to be more probable on the assumption that some kind of agent adjusted the fundamental constants or fiddled with molecules in the pre-biotic soup, than it is on the assumption that these processes were just random. $^{8}$

The following kind of response to the Preference Problem may be tempting. Suppose we stipulate that the kind of agent we are positing to explain 
the existence of life is one that values life and would do what is necessary for the universe to contain living creatures. The existence of life certainly is more likely on the assumption of this stronger bias-hypothesis, than on the Chance hypothesis. So it seems that this hypothesis is confirmed by the existence of life. And since this hypothesis is one in which the processes that resulted in life were biased, this might seem to raise doubts about the Chance Hypothesis.

That this response is misguided should be clear from the fact that no matter how the universe had turned out, an argument along these lines would be available. Whatever the values of the fundamental constants were, we could posit a being whose intention it was to make a universe with just those constants, and argue that since this hypothesis is confirmed, we have evidence against the Chance Hypothesis. But it cannot be that the same conclusion could be reached from the way the universe is, no matter what that way is.

The error may be illustrated by an analogy. Upon winning the lottery I note that my chances of winning if the lottery was fair (the Chance Hypothesis) were one in a million. So I suspect that the lottery was rigged (i.e., that the ticket selection was intentionally biased). Obviously I am confused. The assumption merely that the lottery was rigged renders it no more likely that $I$ should win, for there are many people for whom it could be rigged, and it is no more likely to be rigged for me than for anyone else. It will not help to appeal to the stronger rigging hypothesis, namely that the lottery was rigged for me. Sure, this stronger hypothesis does make my winning more likely and hence is confirmed by my winning. But even upon confirmation, this stronger hypothesis can be no more probable than the weaker hypothesis simply that the lottery was rigged (this follows from the theorem that if $\mathrm{P}$ entails $\mathrm{Q}$, then $\mathrm{P}(\mathrm{P} \mid \mathrm{K}) \leq \mathrm{P}(\mathrm{Q} \mid \mathrm{K})$, for any background knowledge $\mathrm{K})$. What we have here is a counter-example to the deceptively appealing principle that if $\mathrm{P}$ confirms $\mathrm{Q}$ and $\mathrm{Q}$ entails $\mathrm{R}$, then $\mathrm{P}$ confirms $\mathrm{R}$. If confirmation is understood as raising of probability, then my winning the lottery confirms - to some negligible degree - that the lottery was rigged for me without confirming that the lottery was rigged. Similarly, although the hypothesis that there was intentional biasing for life is confirmed to some degree by the existence of life, it does not follow that life's existence confirms the weaker intentional biasing hypothesis at all, and hence it does not follow that life's existence gives us any reason to doubt the Chance Hypothesis.

While the Preference Problem poses a legitimate challenge for anyone who wishes to attribute life's emergence to the work of a rational agent, we should note that it is far from devastating. The main problem is that it appears to prove far too much. If we really are completely clueless as to the likely preferences of an agent capable of creating life, then there is nothing even in principle that such an agent could do to give us any evidence for its existence. For the only way that data can raise the probability of a hypothesis is if those data are more to be expected assuming the truth of this hypothesis than 
assuming the alternatives. But this cannot be right. It is not hard to think of hypothetical cases in which we should be inclined to think there is such a being regardless of our initial skepticism. David Hume (1935) imagines hearing a melodious voice from the sky, speaking a profound message in every language to every part of the globe at once. Such sounds could come about by the random motion of air molecules. But were we to hear it we would have little doubt that some kind of agent was at work. My favorite example of this sort comes from John Leslie (1989). Powerful microscopes reveal the words "MADE BY GOD" in tiny letters on every inch of matter in the universe. In such a case we couldn't help but suspect that an agent was responsible, even if we are baffled as to how this could be done. But if, as the proponent of the Preference Problem claims, we can simply have no idea as to the likely preferences of a supernatural agent, then we have no reason at all to suspect that such an agent was responsible for these words.

Of course whether or not life's existence is like the voice from the clouds or microscopic molecular messages in this respect is another matter. But we can see how the debate could proceed. What is it about the microscopic words that make it seem obvious that they were put there on purpose? This pattern must somehow stand out among the possibilities as the kind of thing that a rational agent might be sensitive to, even if we know very little about such an agent. There is room then to argue that the same holds in the case of life's existence. But to pursue this matter further would take us too far a field, as it is not speculations about intentional biasing which scientists typically appeal to when rejecting the Chance Hypothesis for life's origin.

\section{Non-intentional Biasing for Life?}

Suppose then that we are not going to appeal to the possibility of intentional biasing. Is there any other reason to doubt the Chance Hypothesis, which involves only non-intentional considerations? That is, is life more to be expected on the assumption that the processes from which it resulted were non-intentionally biased, than on the Chance Hypothesis? Let's consider the cosmological and biological cases in turn.

\subsection{The Cosmological Case}

Some philosophers (e.g., Smart (1989)) who have been struck by the so-called fine-tuning of the universe for life, have suggested that the way to resolve this apparent puzzle may lie in the discovery of more fundamental laws which constrain the values of the constants on which the existence of life depends. Now some scientists apparently think that we should expect to discover such laws entirely independently of any puzzle about life. That is, even if it was known that just about any way the big bang turned out could have supported life, they would still find it dissatisfying that there are so many features of the universe that take arbitrary values, ones which cannot be deduced from 
a more fundamental theory (see, e.g. Weinberg 1992). They think that we should expect to find some very elegant theory with few free parameters from which everything else can be deduced. I am not sure what to make of this attitude, but let's be clear that this is not the view that concerns us here. What interests me is the suggestion that the fact that the actual values of the fundamental constants (or ones close to them) are necessary for life, is a reason to suspect that there are more fundamental laws constraining these constants.

It strikes me that while the Preference Problem against the suggestion of intentional biasing is debatable, the parallel objection to the current suggestion is devastating. Let us suppose that there do exist more fundamental laws that constrain the physical constants to some unspecified narrow range of values. There seems to be no conceivable reason to suppose that these laws are more likely to constrain the constants to some particular set of values over any others. So the probability that life will exist given that there are more fundamental laws, is no greater than on the Chance Hypothesis. Certainly if we just focus on the values of the constants themselves, there is nothing about these actual values which makes them stand out as the kind of values that fundamental laws are likely favor. Does the fact that certain values are necessary for life make them more likely to be favored by laws? I can't imagine why anyone would think so. While there is at least room to argue that a rational agent is likely to influence the physical parameters in order to allow for the evolution of life, to suppose that impersonal physical laws are likely to constrain the constants in this way can only be based on a confused anthropomorphism. What makes the particular narrow range in which the actual values of the physical constants fall stand out among the full range of apparent possibilities, is that just that they permit the existence of something that strikes us as special or interesting or valuable, namely life. Even if this value we attach to life is something objective, whether it be moral or aesthetic, or whatever, it could only conceivably have influence on the behavior of an agent. Blind physical laws are no more naturally drawn toward states of affairs with value than blind chance is.

It would be a mistake of course, to try appealing not merely to the hypothesis that there are some fundamental laws which constrain the constants to some values but to the stronger hypothesis that they are constrained to their actual values, or at least to the range of life-permitting values. This commits the same mistake as the response to the Preference Problem with respect to intentional biasing considered above. No matter how the big bang turned out, we could posit a set of laws that constrain the values of the constants to the resulting values. Such a hypothesis would be confirmed to some negligible degree, but the hypothesis that there are any laws at all governing the values of the constants need not be confirmed one iota. The mistake here would be similar to me having won the lottery and speculating not that an agent rigged it for me, but that there is some law or impersonal physical cause that made it likely that I would win all along. The problem is not just that the existence 
of such a law is implausible. The more important point is that as far as we can tell, such a law would be no more likely to work in my favor rather than any other ticket holder.

\subsection{The Biological Case}

Somewhat surprisingly, the very same conclusions seem to apply to the case of the molecular origins of life. Of all the ways that molecules can fall together by chance, we are told, an extraordinarily small proportion constitute the kind of self-replication machinery required for a process of natural selection to get going and lead to life as we know it. So the chance of life arising by chance, even given the basic organic chemical ingredients and a hospitable environment, is said to be incredibly low. Now let's suppose that the process by which these complex molecules arose was not just a matter of chance, but rather was (non-intentionally) biased towards certain molecular configurations. Are self-replicating, life-producing molecules more likely to appear on this assumption? I am unable to see any reason to think so. We can think up any number of ways that the process could be biased. We can speculate about a range of possible laws and physical conditions such that simple atoms and molecules tend to cluster in certain ways rather than others. Some of these may favor life's emergence; others will disfavor it. As in the cosmological case, what makes certain molecular configurations stand out from the multitude of possibilities seems to be that they are capable of developing into something which strikes us as rather marvelous, namely a world of living creatures. But there is no conceivable reason that blind forces of nature or physical attributes should be biased toward the marvelous.

Where does this leave us? If life's existence is no more to be expected on the assumptions of either intentional or non-intentional biasing than it is on chance, then we have no reason to doubt the Chance hypothesis. I have been arguing that while there is at least room to argue that life is more to be expected given that an agent was involved, it is very hard to see why we should find life's existence any more likely at all on the assumption that nonintentional biasing factors were involved. So unless we suspect that life arose on purpose, we should be quite content to join Crick in seeing life as an extremely improbable "happy accident".

\section{Clarification and Analogies}

I would like to be as clear as possible about what I am suggesting by first being explicit about what I am not suggesting. First of all, I am not suggesting that the search for non-chancy, non-intentional kind of explanation for life's emergence is misguided because there could not be such an explanation. For all that I have said, there could be a perfectly satisfying explanation of life's origin that appeals neither to blind chance nor to agency. There might, for instance, be some basic laws that determine that the fundamental 
constants cannot differ from their actual values. There might be something comparable at the molecular level applied to the formation of a first selfreplicator. Knowledge of such facts would help us understand why life exists, and this would be a very interesting discovery.

The worry that I am raising concerns only whether the phenomenon of life gives us any reason at all to expect that there are such explanatory facts to be discovered. Compare again the lottery example. It might well be that the lottery mechanism is so constructed that no matter how the different tickets are tossed around in the barrel, ticket \#48579387593478 is very likely to be picked out. Perhaps there is a trace of iron in that ticket, and a powerful magnet in the motor pulls it toward one corner. Discovering this might be interesting, as it would help us understand why, out of all the millions of tickets, this one was selected. Nevertheless, it is thoroughly misguided to think that ticket \#48579387593478's being selected gives us any reason at all to suspect that an explanation of this sort is available. No matter how confident or doubtful we are initially that the lottery is fair in that tickets are selected purely by chance, ticket \#48579387593478's being selected gives us no reason at all to doubt this. Any investigation into the lottery mechanism that was motivated by the fact that ticket \#48579387593478 was selected would be misguided.

Secondly, nothing that I have argued challenges the credibility of any extant proposal concerning the emergence of life. For all that I have argued, one of the current scientific speculations that appeals neither to chance nor agency may be correct, and its proponents may be justified in endorsing it. Of course this is a field in which there is deep disagreement, with experts convinced that their colleagues are entirely on the wrong track. So the nonspecialist is in no position to know what to make of it. But there is nothing to rule out someone's having discovered that life's emergence was to be expected given certain laws and conditions, just as there is nothing to rule out our discovering that a lottery mechanism is biased.

The following two stories should help illustrate case I have been making. In the first one we are playing Bridge and Fred deals himself a hand of thirteen spades. This might strike us as a little suspicious, leading us to suspect that the dealing was biased in some way. Just how strong a reason we have to suspect that an agent such as Fred biased the dealing may be controversial; it will depend on various factors such as how strong an incentive he would have given the stakes and how difficult it would be to pull it off. So it is not clear whether we have much reason to suspect that dealing was intentionally biased rather than just a matter chance. But it would be thoroughly wrong-headed to respond as follows:

I doubt that those hands were dealt by an ordinary random shuffling, where each card has about an equal chance of landing in any position in the pack. But rather than suspecting Fred or any other agent of foul play (since Fred 
couldn't and wouldn't do it, and I don't believe in supernatural agents), there is probably an explanation involving impersonal physical mechanisms. Perhaps there are attractive forces between the cards that cause them to cluster in certain configurations during a normal shuffling, and these tend to favor all spades being dealt to the dealer.

The problem with this suggestion is not the implausibility of the theory; we can pretend that it has as much initial plausibility as we like (perhaps we are playing with a unusual set of cards whose properties are largely unknown to us). The problem is that on the assumption that there are such cardclustering forces, they are no more likely to favor certain orderings over any others (unless we suspect that these forces favor certain orderings because someone intended the cards to cluster in certain ways). So the fact that Fred was dealt all spades gives us no reason to suspect that such a theory is correct, or indeed any theory that does not involve rational agency. The point is that the only thing that makes a hand of spades stand out among the possible hands as calling for a non-chancy explanation, is that the hand is one that may have significance for a rational agent. Fred's lucky hand should therefore either suggest that there is some intentional explanation, or be dismissed as a "happy accident".

In the second story a meteorite shower results in millions of little rocks arranged in the Australian desert to form a proof of Goldbach's Conjecture. I doubt that anyone in this case would be comfortable with saying that the rocks just fell this way by chance. Whatever our prior convictions, we couldn't help but suspect that an agent somehow directed the course of the rocks even if we can't imagine how. The following response however, would be confused:

There is no doubt that this didn't just happen by chance alone. The probability of randomly falling rocks landing in such a pattern is just too low! But let's not jump to any conclusions about intelligent agency. This puzzling state of affairs should motivate a new scientific research program to discover the nonrandom physical mechanisms that govern the way rocks fall from space given the appropriate conditions. We might not have the answer yet, and the current suggestions may be only sketchy, but we have every reason to expect that the correct explanation is of this sort.

One difference between the two stories is that in the first it is not obvious how much, if at all, we should doubt the Chance Hypothesis. In the second, the Chance Hypothesis seems incredible. Whether the case of life's existence is closer to the first or the second story in this respect is not a question that I have tried to address. This is what I am suggesting both stories have in common with the case of life: We have a state of affairs which is highly improbable on the Chance Hypothesis, but is no more to be expected given that some kind of non-intentional biasing was involved. The denial of the Chance hypothesis raises the probability of this state of affairs only if the 
intentional biasing hypothesis does. Yet while making no appeal to intentional agency, researchers are persuaded that this was not the result of chance, and are motivated to find a non-intentional explanation. While the reasoning in the case of life might not seem as misguided as in the stories, our discussion thus far suggests that it is.

\section{Complexity}

As my conclusion is at odds with respected scientific opinion, it is worth trying to discern where our reasoning might have gone wrong. One line of response that I've encountered is that it is not strictly life's existence which could not plausibly have arisen by chance, but rather the extraordinary complexity of the structures required for life. What has struck scientists with such awe is that even the very simplest cell is an enormously complicated piece of machinery, more intricate and complex than any machine made by humans. No doubt life began in a somewhat simpler form, but it is widely held that the kind of systems required for a process of natural selection to get going would also have to be extraordinarily intricate and complex. It might seem that this alone is what stands in need of explanation, whether or not the machinery happens to be living or life-producing.

Just what it is for something to be complex in the relevant sense is rarely explained very well, but it is generally acknowledged that the idea is well captured by Fred Hoyle's (1981) suggestion that the random assembly of the very simplest living system would be like a tornado blowing through a junkyard and assembling a Boeing 747 (Dawkins 1987 and de Duve 1995 focus on this example in part to illustrate the alleged absurdity of attributing the emergence of life to chance). Obviously part of what makes something complex in this sense is that it has a heterogeneous structure, being made up of very many parts of various shapes and sizes. But any pile of 747 parts meets this condition. Furthermore, that the 747 parts should be randomly assembled into a jumbled pile of some very specific shape and structure is just as improbable as their being assembled into a plane. What then is the significant difference between the pile of 747 parts and the 747 ? The idea seems to be what Hume (1935) described as "the curious adapting of means to ends" (p. 34) Like a living system, the plane consists of very many parts working intricately together to perform a function, namely flying. The parts require a very specific arrangement for this to work; if any one part is in a slightly different position, then the plane can't perform its function. The pile of plane parts on the other hand don't do anything but sit there, or topple over if you push them enough. You don't need a very precise arrangement of parts to do that, so there is nothing very remarkable about such a pile forming by chance.

On a closer look however, this apparent difference is not so deep. For any pile of plane parts we could define a very specific functional property taking the form: the pile is such that when this part is pulled in precisely 
this direction, precisely this far, then the pile will topple into this very specific structure.... This functional property requires for its instantiation an extremely precise arrangement of parts; shift one part and the pile will not have exactly the same toppling tendencies. Call a pile of plane parts which has this functional property a "schmane" What planes and schmanes have in common is that the probability that tornado strewn plane parts would assemble either is extremely low. Why then are we so resistant to the idea that a tornado might assemble the parts into a plane, but have no trouble supposing that it might produce a schmane? The answer should be that a tornado is just as likely to produce either, it is just that only the plane is more likely to result if there was more than just chance operating. But now we must return to the question of whether it is the assumption of intentional or non-intentional biasing which renders this outcome more probable. Certainly the plane might seem more likely on the assumption that an agent influenced the arrangement of the parts (that is why if we found one on a distant planet we would conclude that extraterrestrials had built it, even if we had never seen a plane before, and even if our theories made the existence of such creatures in the vicinity very unlikely). But it is hard to see any reason to suppose that on the assumption of non-intentional biasing, a plane is any more likely than a schmane. Any considerations which make planes stand out as special as compared to schmanes, are intentionally related - whatever intuitions we have about the case have to do with what we think an agent is likely to do.

We seem to be in the same situation with respect to the molecular machinery from which complex life forms developed. These molecules are intricate little machines that perform a certain functions, most important of which is the assembly of new machines identical to themselves with a high degree of accuracy. But for any large heterogeneous aggregate of molecules we can define some very specific functional property $\mathrm{F}$ that it possesses, such that it is extremely unlikely that a random assembly of molecules will result in something with property $F$. Yet for the vast majority of such properties, no one will have any trouble believing that it was just a matter of chance that some molecules were arranged this way. What makes the complex macromolecules from which life developed so special? Unlike just any arbitrary function, their ability to create replicas of themselves strikes many as crying out for a nonchancy explanation. But once again we may have a sense as to why an agent might be inclined to form such a molecular structure rather than others. But it is hard to imagine why what de Duve calls the "combinatorial properties of matter" if they favor particular molecular configurations at all, should be biased toward those capable of self-replication.

\section{De Duve's Defense}

It is worth considering a brief argument by Christian de Duve (1995), as it is the only case that I am aware of in which a distinguished scientist explicitly 
defends the widely held position that I am challenging. De Duve anticipates the objection that highly improbable events are commonplace. He notes for example that each particular distribution of cards among players in a Bridge game has a probability of about one in fifty billion billion billion. Yet when most Bridge hands give us no reason to doubt that the cards were dealt randomly. To illustrate why the case of life's origin should be treated very differently, de Duve first appeals to Hoyle's analogy of the Boeing 747 arising ready to fly from the tornado-swept junkyard. The implication is apparently that it would be absurd to suppose that this happened just by chance. Already this is an odd analogy to appeal to, for our intuitions in this case are clearly driven by the fact that a 747 appears to be the work of an intelligent agent. Yet this is not at all the kind of explanation that de Duve has in mind for the case of life.

According to de Duve, the flaw in the Bridge analogy is that in the case of life's origin we are not dealing with just a single event. For there are many successive conditions that must be met, and many diverse parts assembled in order for life to exist. According to de Duve,

This consideration completely alters the probability assessment. We are being dealt thirteen spades not once but thousands of times in succession! This is utterly impossible, unless the deck is doctored. (1995: 9)

De Duve concludes that there must be some brute physical constraints ensuring that the many necessary steps to life each had a very high likelihood of obtaining.

However, it is very difficult to see how the number of independent events involved could make a crucial difference in these cases. The outcome of a single dealing session could be seen as depending on very many improbable events if we include every neuron-firing and minute muscle contraction of the card shuffler on which the final distribution of cards crucially depends. And conversely, we should be no less astonished at obtaining thousands of thirteen-spade hands from a single selection from an enormous deck. The number of events involved seems beside the point.

What is striking about the selection of only spades is the simple regularity displayed. The same salient property is instantiated over and over without exception. This is what is implausibly attributed to blind chance. As was mentioned in connection with the size-ordered pebbles on the beach, the discovery of widespread correlations between salient properties may well suggest that some non-chancy but non-intentional explanation is correct. For on the assumption that some kind of non-intentional biasing was involved, say by various causal connections limiting the range of ways that these properties can be distributed, then some kind of regularity among properties is more to be expected. 
But the numerous steps required for life to exist are quite unlike this. It is not a matter of the same event-type or property being instantiated many times without exception. The conditions required for the emergence of life have little at all in common. The picture given by de Duve and others is more like the following. We require one kind of chemical to be present, plus another very different one, and yet another different one, and we require the absence of still other substances. Certain chemical reactions must take place, then others involving different ingredients and producing different outcomes. A wide variety of events that would undermine the whole process must fail to occur. The molecular parts required to make up the replication machinery come in various sizes and structures. And they are not arranged in anything like a simple repetitive pattern but rather each has a very unique position and role to play. Rather than numerous hands of only spades, a more apt analogy would be being dealt a large variety of thirteen-card hands with no salient features in common.

Of course while intrinsically the various conditions required for life may be very diverse, they do share a certain feature in common: They are all requirements for life's existence. Could the fact that these conditions share this feature make it implausible that they came about by chance? This depends on whether it is plausible to suppose that life came about by chance if the probability of this happening was extremely low. Whether we are dealing with numerous conditions whose conjunction is highly improbable, or just one highly improbable condition doesn't appear to make any interesting difference.

To continue the card analogy, suppose we are dealt some disparate collec-

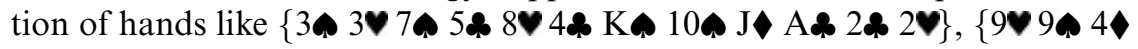

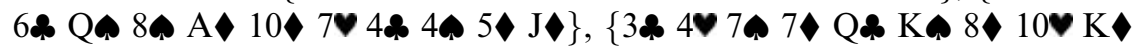

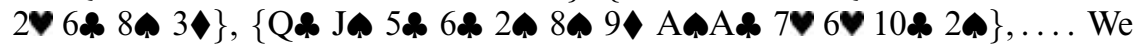
shouldn't be reluctant to suppose this is the result of an ordinary random shuffling and dealing. Of course investigation might reveal that indeed the shuffling mechanism was biased toward this outcome. But there is nothing at all about the sequence as such that should make us doubt that it came about by chance. Now suppose that we have a machine into which we can feed a series of cards whose sequence determines how it functions. It turns out that the vast majority of card-sequences allow the machine to do nothing very interesting, perhaps just jumbling about various parts in one way or another. But if we feed in this very sequence that we have been dealt, the machine does something strikingly different. Let's say it assembles a Boeing 747. In this case we might well come to doubt that these cards were just shuffled randomly. But of course in this case our suspicion would be that an agent had doctored the deck. If an agent were to doctor the deck she is somewhat more likely to arrange for a sequence of cards that is required for the assembly of a 747, rather than just some pile of junk. But if we are considering just impersonal physical laws and properties and the like, it is entirely mysterious 
why we should expect these to favor a sequence required for the production of a 747 over any other outcome.

\section{A Possible Diagnosis}

I've argued that the phenomenon of life gives us no good reason to doubt that it arose by chance, unless we think life's existence is more likely on the assumption of intentional biasing. Why then are most scientists so reluctant to allow too much chance into their accounts of life's emergence? I will offer a speculative diagnosis. The conviction that life couldn't have arisen by chance is typically a gut reaction to the data, not a conclusion arrived at on the basis of a theory about when it is plausible to ascribe something to the work of chance. When Dawkins insists that no one can sensibly think that something as complex as an eye, or DNA/protein replication machinery could spontaneously emerge in a single chance event, he expects us to just find this obvious. When we look at the extraordinary complexity of the mechanisms involved, and the way that so many intricate parts work together to perform specific tasks, and the extreme improbability of a chance jumbling of parts forming something which functions in anything like the same way, it just strikes most people as absurd that this could have happened by chance.

Now consider these thought-experiments. As we have noted above, even people extremely skeptical of any supernatural intelligence can describe possible situations where they would suspect that some agent with powers well beyond ours was at work (e.g. Hume's voice in the sky, Leslie's microscopic messages). Here is one that everyone I've talked to has found compelling. Tons of Lego pieces are tossed off the top balcony of the Empire State Building. Watching below we see them fall and bounce and blow about in the wind, and form themselves into an enormously complex mechanism, say a scale model of a watch, or a Boeing 747, or a human eye. Of course if we just found such a Lego machine already assembled, we would have no doubt that a team of humans had assembled it. But in the case described this explanation seems all but ruled out, as humans just don't have the capacity to control the motion of millions small pieces as they are thrown about. But everyone who contemplates this case has a hard time not believing that there was some kind of agent at work. As far as my diagnosis goes, it is not important whether this reaction is reasonable; it is just the reaction that everyone has. Furthermore, it is not crucial that the Lego mechanism so formed is of a kind with which we are familiar. Our reaction would be the same even if we had never seen or imagined a watch or plane. So suppose now the Lego pieces fell and formed an exact working scale model of DNA/protein replication machinery. I take it that most people would react exactly the same way, if not with even greater conviction that some agent was responsible, regardless of their initial skepticism about agents with super-human powers. 
Now I can't imagine why the size of the mechanism should make a difference to our intuitions. This suggests that the intuition that people really have when they contemplate the molecular mechanisms from which life developed is that they look as though they were designed by an agent. Perhaps this is why it strikes people as extraordinary that life arose by chance. From the conviction that life did not arise by chance, scientists are then led to seek an explanation for life's origin which in no way appeals to an agent's intentions. So the line of reasoning, if made more explicit is something like the following. That molecular replicating systems appear to be designed by an agent is sufficient to convince us that they didn't arise by chance. But in scientific reasoning, non-intentional explanations are to be preferred, if possible (some would say at all costs), to intentional ones-hence the motivation to find a non-intentional explanation of life.

It should be clear however, that even granting the appropriateness of a preference for non-intentional explanations, this line of reasoning is confused. In general, if $B_{I}$ raises the likelihood of $S$, then $S$ confirms $B_{I}$ to at least some degree, and may thereby disconfirm $\mathrm{C}$. But it does not follow that $\mathrm{S}$ confirms $\mathrm{B}_{\mathrm{N}}$ one iota. $\mathrm{S}$ confirms $\mathrm{B}_{\mathrm{N}}$ only if $\mathrm{B}_{\mathrm{N}}$ raises the likelihood of $\mathrm{S}$. If the reason we doubt the Chance Hypothesis is that we suspect that life is due in part to intelligent agency, this by itself gives us no reason to expect there to be a non-intentional explanation for life. If on reflection we do not find the hypothesis of intentional biasing acceptable, then we are left with no reason at all to doubt that life arose by chance.

\section{Conclusion}

My argument has been that the following combination of attitudes is misguided:

(i) Even if we do yet have an adequate explanation of life's emergence, knowledge of the physical, chemical, and biological conditions that life requires makes it extremely implausible that life simply arose by chance. Rather, we have reason to think that there is something about the relevant physical properties, forces, laws, and other conditions (having nothing to do with the purposes of any agent) which make it rather likely that life should come into being.

This leaves open at least the following options, among which I haven't attempted to adjudicate.

(ii) There is a non-intentional explanation for life's existence, one that makes a more limited appeal to chance, or perhaps leaves nothing up to chance at all. But our reason for thinking so is that a specific proposal has been investigated and confirmed to some degree; it is not that we just think there must be such an explanation, given the requirements for life's existence. 
(iii) Life's emergence was due, at least in part, to the work of an agent.

(iv) Life's emergence is the extremely improbable result of chance.

I am not entirely comfortable with this conclusion, given that (i) is a very common position among scientists working in fields relevant to the origin of life. There may be some grounds for (i) that I have failed to appreciate. But at the very least, the considerations raised here should encourage us to rethink the assumptions at work in origins of life research. ${ }^{9}$

\section{Notes}

${ }^{1}$ I discuss the notion of explanatory stability in more depth in White 2005.

${ }^{2}$ See Barrow J. and Tipler F. (1986), and Leslie (1989) for surveys of the data supporting these claims.

${ }^{3}$ Robert Shapiro (1986) provides a marvelously readable survey of contemporary origin of life theories, all of which he vigorously argues are untenable. But he too cannot bring himself to accept that life's emergence was a radically chancy affair, and is confident that there must be some less chancy explanation.

${ }^{4}$ Fry's two complaints against the "Almost Miracle Camp" are first that it puts an end to scientific research into life's origins and second that it leaves the way open to explanations involving agency, such as Hoyle and Wickramasinghe (1981).

${ }^{5}$ Fry includes Crick in this camp. But it is not clear how comfortable Crick is with this view, since in the same book he speculates about living molecules perhaps being delivered to Earth by extraterrestrial creatures.

${ }^{6}$ Dawkins suggests that if there are $\mathrm{N}$ possible opportunities for the spontaneous random generation of a replicator to occur, then the largest "ration of chance" that we should allow in a serious theory is one that assigns a chance of at least $1 / \mathrm{N}$ for spontaneous generation on any particular occasion. So on Dawkins' account the lowest chance that we can postulate for the spontaneous generation of life at some time is $1-(1-1 / \mathrm{N})^{\mathrm{N}}$, which is greater that 0.6 for any value of N. In other words, according to Dawkins account, any acceptable theory will say that life's emergence at some time was at least more likely than not.

${ }^{7}$ Gould (1990), Katz (1988) and Scriven (1969) seem to have fallen into this confusion, which van Inwagen calls "the most annoyingly obtuse argument in philosophy" (1993:67).

${ }^{8}$ See Manson (2000) and Sober (2003) for discussions of objections along these lines.

${ }^{9}$ This paper arose from questions first posed to me by Tom Nagel, and I've benefited a lot from our continued discussions. Thanks also to Adam Elga, Pete Graham, Ned Hall, Peter Kung, Chris Peacocke, Susanna Rinard, and Bob Stalnaker for feedback, none of whom should be held responsible. Some of this material was presented at colloquia at CUNY Graduate Center and at NYU. I'm grateful for the feedback I received on those occasions.

\section{REFERENCES}

Barrow J. D. and Tippler, F. J. (1986) The Anthropic Cosmological Principle. Oxford: Clarendon Press.

Crick, Francis. (1981) Life Itself: Its Origin and Nature. New York: Simon and Schuster.

Dawkins, Richard. (1987) The Blind Watchmaker. New York: Norton.

De Duve, C. (1991) Blueprint for a Cell: The Nature and Origin of Life. Burlington NC: N. Patterson. 
( 1995) Vital Dust. New York: Basic Books.

Eigen, M. (1992) Steps towards Life. Oxford: Oxford University Press.

Fry, Iris. (1995. "Are the Different Hypotheses on the Emergence of Life as Different as They Seem?" Biology and Philosophy 10(4): 389-417.

- (2000) The Emergence of Life on Earth: a Historical and Scientific Overview. New Brunswick, NJ: Rutgers University Press.

Gould, S. J. (1990) "Mind and Supermind" in Physical Cosmology and Philosophy, John Leslie, ed. Englewood: Prentice Hall.

Hoyle, F and Wickramasinghe, C. (1981) Evolution from Space. London: Dent.

Hume, David. (1935) Dialogues Concerning Natural Religion, Norman Kemp Smith, ed. Oxford: Oxford University Press.

Katz, Jonathan. (1988) "Why there is Something rather than Nothing: The Anthropic Principle and Improbable Events", Dialogue 27: 111-120.

Leslie, John. (1989) Universes. London: Routledge.

Manson, Neil. (2000) "Anthropomorphism and the Design Argument" Religious Studies 36: $163-76$.

Mayr, Ernst. (1976) Evolution and the Diversity of Life. Cambridge: Belknap Press.

Monod, Jacques. (1971) Chance and Necessity: An Essay on the Natural Philosophy of Modern Biology. New York: Knopf.

Scriven, Michael. (1969) Primary Philosophy. New York: McGraw-Hill.

Shapiro, Robert. (1986) Origins: A Skeptics guide to the Creation of Life on Earth. New York: Summit Books.

Smart, J. J. C. (1989) Our Place in the Universe: A Metaphysical Discussion. Oxford: Blackwell.

Sober, Elliot. (2003) "The Design Argument", in God and Design: The Teleological Argument and Modern Science, Neil A. Manson, ed. London: Routledge.

van Inwagen, Peter. (1993) Metaphysics. Boulder: Westview Press.

Weinberg, Stephen. (1992) Dreams of a Final Theory: The Scientist's Search for the Ultimate Laws of Nature. New York: Pantheon Books.

White, Roger. (2005) "Explanation as a Guide to Induction" Philosophers' Imprint 5(2). 